\title{
Videotutoriales y códigos QR: recursos TIC en laboratorios de Ciencias de la Comunicación
}

\author{
Videotutorials and QR codes: ICT resources at Communication Sciences \\ audiovisual labs
}

\author{
Carmen del Rocío Monedero Morales ${ }^{1}$, Antonio Castro Higueras² ${ }^{2}$ Juan Tomás Luengo \\ Benedicto $^{3}$ \\ ${ }^{1}$ Departamento de Periodismo, Universidad de Málaga, España (roi@uma.es) \\ ${ }^{2}$ Departamento de Comunicación Audiovisual, Universidad de Málaga, España (acastro@uma.es) \\ ${ }^{3}$ Departamento de Periodismo, Universidad de Málaga, España (jtluengo@uma.es)
}

Recibido el 23 de enero de 2017; revisado el 5 de septiembre de 2017; aceptado el 2 de noviembre de 2017; publicado el 2 de diciembre de 2017

\section{RESUMEN:}

El uso de las tecnologías de la información y la comunicación (TIC), como herramientas de enseñanza, se ha consolidado como una alternativa dentro y fuera del aula que permite el desarrollo de procesos de aprendizaje significativo y colaborativo y en los que se da una mayor interacción entre el estudiante y el objeto de estudio. A partir de esta premisa, un grupo de profesores de la Facultad de Ciencias de la Comunicación de la Universidad de Málaga han puesto en marcha un proyecto de innovación educativa consistente en la realización de videotutoriales sobre los distintos equipos presentes en los laboratorios audiovisuales, así como de ciertas habilidades comunicativas generales útiles para la superación de asignaturas enmarcadas en los grados de Periodismo, de Publicidad y Relaciones Públicas y en Comunicación Audiovisual. Tras la realización, bajo la supervisión del tutor de la materia, de los videotutoriales por parte de los alumnos se les asociaron códigos QR ubicados en los equipos y en las aulas. Este artículo analiza el proceso de desarrollo de la primera fase del proyecto, así como los resultados de dos cuestionarios de detección de necesidades de aprendizaje enviados a los alumnos y al profesorado de la Facultad, en los que se revelan tanto la demanda de implementación de este tipo de metodologías por parte del alumnado, como la actitud positiva hacia su uso por parte de los docentes.
PALABRAS CLAVE: TECNOLOGÍA DE LA INFORMACIÓN, APRENDIZAJE COOPERATIVO, APRENDIZAJE MIXTO Y COMUNICACIÓN AUDIOVISUAL.
ABSTRACT:
The use of ICT (new Information and Communication Technologies) as teaching tools has established itself as an alternative within and outside the classroom that enables the development of significant and collaborative learning processes, and in which there is greater interaction between the student and the object of study. From this premise, a group of assistant and associate professors from the three degrees taught at Faculty of Communication Sciences at the University of Malaga have launched an innovative educational project involving the realization of videotutorials on different equipment present in audiovisual laboratories and of certain general communication skills useful for overcoming subjects framing in the degrees in Journalism, in Advertising and Public Relations and in Audiovisual Communication. After the execution of videoturials by students, under the supervision of the tutor of every single subject, Quick Response Codes (QR codes) were associated to them and located on every equipment and in all the classrooms. This article analyzes the first development process phase of the project, as well as the results of two different learning 
needs identification surveys sent to Faculty members (students and professors), which reveal on one hand, this type of methodologies implementation demand by the students, and on the other hand, professors positive attitude towards its use.

\begin{tabular}{|c|c|c|}
\hline COOPERATIVE & LEARNING, & BLENDED \\
\hline LEARNING & AND $\quad A$ & AUDIOVISUAL \\
\hline
\end{tabular}

\section{INTRODUCCIÓN}

La sociedad actual, denominada "de la información y el conocimiento" presenta un panorama complejo marcado por el dominio de la tecnología y los mercados globales. El papel de la enseñanza universitaria, redefinido en el marco de la convergencia europea a través de la reforma de Bolonia, busca su adaptación a un entorno diverso, cambiante y competitivo. Este cuestionamiento se ha canalizado haciendo hincapié en dos aspectos básicos: de un lado, crear las condiciones que favorezcan la autonomía del alumno universitario para la adquisición de competencias (en lugar de contenidos), de forma que se aproxime el entorno educativo al ámbito profesional $\mathrm{y}$, de otro, la asimilación de nuevas metodologías y recursos tecnológicos para la enseñanza al amparo de las tecnologías de la información y el conocimiento (TIC).

A partir de este nuevo enfoque surgen multitud de iniciativas fruto del diseño y desarrollo de proyectos de innovación docente. Una de ellas, la que aquí se presenta, se enmarca dentro del proyecto de innovación educativa (en adelante PIE), actualmente en curso, "Recursos tecnológicos para la enseñanza en laboratorios audiovisuales: videotutoriales y códigos QR" (PIE 15-87), subvencionado por la Universidad de Málaga (UMA) durante el bienio 2015-2017.

El proyecto se centra en el uso de una herramienta de innovación docente específica, el videotutorial, en una serie de asignaturas pertenecientes a los tres grados que se imparten en la Facultad de Ciencias de la Comunicación de la UMA (Periodismo, Publicidad y Relaciones Públicas y Comunicación Audiovisual) con el objetivo de reforzar los procesos de adquisición de competencias que han sido desarrolladas previamente en el aula mediante el método de la lección magistral.

La propuesta aquí presentada avanza en la dirección del vídeo, intentando plantear un manual de instrucciones de los distintos equipos presentes en los laboratorios de Ciencias de la Comunicación (cámaras, mesas de edición, cabina de realización, micrófonos, focos, etc.) que supere a los clásicos apuntes por videotutoriales muy sintéticos, siguiendo la tendencia que incide en que los vídeos más vistos no superan los 4 minutos de duración. Estas piezas se pueden visualizar a través de un lector de códigos QR presente en la mayoría de los teléfonos de los estudiantes, tanto de las asignaturas involucradas en el PIE como del resto de las materias impartidas en la Facultad. La disponibilidad de la información será permanente, afectando incluso a las horas de libre uso de los equipos en las cuales no hay ningún profesor guiando a los estudiantes.

A la hora de abordar el presente artículo se ha comprobado que existen numerosos estudios que analizan el uso de TIC tales como las redes sociales, la plataforma Moodle y los denominados "contenidos educativos reutilizables" 1 como instrumentos pedagógicos en el entorno elearning ${ }^{2}$, pero que apenas existen investigaciones centradas en el videotutorial, el vídeo en directo y los códigos QR como complementos para reforzar la comprensión, siendo una excepción el trabajo de Jiménez y Marín. Precisamente estos autores afirman que

la lección magistral podría ser útil, aunque también serían necesarias sesiones o seminarios adicionales de repaso. En este caso, el método de trabajo autónomo también puede servir para lograr este objetivo, a través de actividades de autoevaluación adecuadas y materiales complementarios como pueden ser las herramientas y materiales diseñados en soporte informático y/o en formato multimedia (Jiménez y Marín, 2012, p. 66).

\section{HERRAMIENTAS TECNOLÓGICAS COMO MATERIAL DE APOYO AL DOCENTE}

La expansión del vídeo como soporte de material didáctico no es ninguna novedad, ya en el año 2005 surgen plataformas y servicios web dirigidos a un público generalista que disponen de contenidos de casi cualquier temática. Posteriormente, aparecen alternativas más especializadas que cubren nichos de mercado más reducidos como TutorialLab o TeacherTube para la formación en vídeo (Llanos, 2008). Dentro de las tipologías de vídeo educativo descritas por Cebrián (como se citó en Bravo, 1996), en el caso que nos aborda nos situaremos en una experiencia de vídeos curriculares, es decir, que se 
adaptan expresamente a la programación de las asignaturas implicadas en el proyecto, en contraposición con los vídeos de divulgación cultural, los de carácter científico-técnico y los vídeos para la educación, que pudiendo utilizarse como herramientas didácticas, se diferencian en algunos matices a la hora de ser concebidos.

Esta evolución es el inicio de la confrontación entre las metodologías de formación tradicionales y la aplicación de nuevas herramientas didácticas (Polo, 2010).

García Sans (2008, como se citó en Espuny, González, Lleixá y Gisbert, 2011) se lamenta de que los recelos hacia las redes sociales no solo se traducen en una escasa investigación acerca de su rendimiento educativo, sino que gran parte del profesorado tiene ese mismo sentimiento de rechazo hacia la tecnología utilizada por los estudiantes, puesto que interfiere en el flujo natural de la enseñanza tradicional.

En un intento de romper con esa tendencia, a la hora de engendrar el proyecto que nos ocupa, partimos de la base de que el uso activo, continuo y social de Internet y de las TIC no debía ser ignorado a la hora de elaborar las planificaciones docentes. Aunque el objetivo del mismo no sea, ni mucho menos, desarrollar el concepto de "escuela virtual" 3 aplicado al ámbito universitario, sino más bien el diseño de materiales multimedia que permitan procesos de aprendizaje complementarios, más flexibles y controlables por el alumno,

teniendo en cuenta que conseguir que los alumnos adquieran conocimientos sobre una materia requiere de un tiempo que va más allá del que discurre en el aula, el uso de las TIC para el diseño de materiales multimedia está teniendo un peso cada vez mayor en los procesos educativos universitarios (Jiménez y Marín, 2012, p. 66).

En esta misma línea se manifiestan López, Ábalos y Espejo (2012, p. 207) para quienes las TIC están motivando una "revolución de la cultura docente, propiciada sobre todo por la importancia de una comunicación interactiva, lo que debe obligar a una revisión de las estrategias docentes utilizadas hasta la fecha".

El uso de los recursos tecnológicos virtuales como herramienta pedagógica no reduce el papel del profesor, sino que, por el contrario, debe "amplificar y empoderar la presencia docente, potenciando las ayudas sociales típicamente ofrecidas por éste en su papel de mediador del proceso de construcción colectiva del conocimiento" (Onrubia, 2005, p. 10).
Aunque este autor advierte que la simple incorporación de las TIC a las prácticas educativas no garantiza la consecución de estos objetivos y anima a mantener y activar la reflexión sobre los objetivos y criterios que deben guiar su inclusión en estas, así como la investigación empírica sobre los resultados y aportaciones de dicha incorporación a la mejora de la calidad de la enseñanza.

Brazuelo y Cacheiro (2010) señalan que los alumnos son los auténticos protagonistas de estos cambios ya que fuera del aula invierten mucho tiempo en estar conectados a las redes sociales. Vinculada estrechamente a la utilización de estas, la telefonía móvil constituye un mercado en constante evolución y en la actualidad, la extensión de la telefonía móvil 4G posibilita la continua conexión a Internet, así como el uso de las herramientas tecnológicas en cualquier lugar y sin necesidad de un ordenador personal. De ahí la importancia del uso de los códigos QR como complemento a los videotutoriales en los que se basa el presente proyecto. Por otro lado, frente al podcast de vídeo se ha empleado el vídeo en streaming, a través de un canal creado al efecto en la plataforma YouTube, eliminado la necesidad de descarga y facilitando el visionado en $P D A$, tabletas, teléfonos inteligentes, etc.

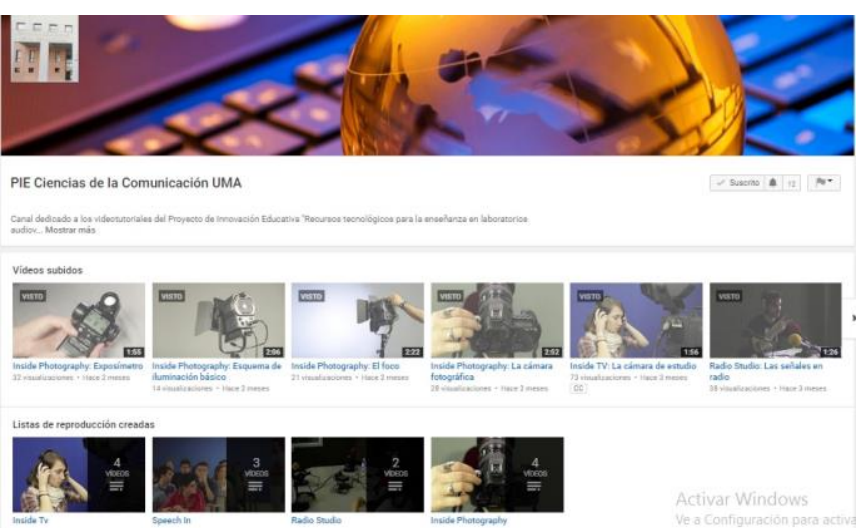

Figura 1. Canal de YouTube donde se alojan los videotutoriales creados por los alumnos

Nos hallamos ante la elaboración, por parte del propio alumnado y bajo la supervisión y guía del docente, de los denominados contenidos educativos reutilizables que, atendiendo a las palabras de Mauri, Onrubia, Coll y Colomina (2005, p. 2) "se presentan en formato multimedia o hipermedia y cuya utilización se prevé que sea posible en situaciones en que el grado de coincidencia física, espacial y temporal de profesores y alumnos pueda darse en un grado mínimo o incluso no existir" ${ }^{\text {" }}$. Zamora y Bello (2011, p. 30) describen el nuevo papel del profesor como el de coach y mentor, debiendo reconocer y utilizar todos los recursos que tiene a su disposición: 
"los ejes de coordenadas cambian y ahora nos movemos entre la posibilidad de comunicación a través de las TIC y su proyección en la enseñanza universitaria". Para ello no hay que limitarse solo a las nuevas modalidades organizativas y métodos de enseñanza sino hay que abrirse a la formación basada en la red (Cabero y Gisbert, 2005), con la integración de herramientas de comunicación sincrónicas y diacrónicas.

El aprendizaje multimedia permite al usuario adquirir los contenidos de forma más eficaz a través de la combinación de información visual y auditiva (imágenes, sonido y texto, la mayoría de las veces en forma de subtítulos o anotaciones) en un único formato de presentación (Mayer, 2001). Entre los materiales multimedia, Mackey y Ho (2008) consideran al videotutorial como un recurso didáctico apropiado para mejorar la absorción de conocimientos, el aprendizaje percibido por el alumno y crear sinergias con otros recursos y métodos de enseñanza. Solo la presencia física del formador aumentaría la calidad de la enseñanza, problema que según Greene (2007) quedará resuelto con la llegada del 3D como siguiente evolución del vídeo, y en último término con los vídeos holográficos de alta calidad. Aunque consideramos que la emotividad, interacción y humanidad producida por la presencia física será insustituible.

Para concluir este apartado creemos interesante poner de relieve que el éxito en el aprendizaje multimedia puede estar condicionado en gran medida por la aceptación de este tipo de tecnologías por parte del alumno. El modelo MAT (modelo de aceptación tecnológica) establece un efecto directo y positivo entre las actitudes hacia el uso, la intención de uso y el propio uso que el individuo hace de la tecnología. A su vez, la utilidad percibida influye en el uso a través de la actitud y la intención de uso (Jiménez y Marín, 2012). Para poder valorar este factor en los resultados de la experiencia aquí descrita, como se explicará más adelante, se facilitó un cuestionario en línea tanto para alumnos como para docentes para tener constancia de este aspecto entre otros.

\subsection{Del aprendizaje cooperativo al aprendizaje mixto}

La clase magistral ha venido siendo el método más extendido en la educación a lo largo de la historia, autores como Fernández (2007, p. 18) afirman que

todos los métodos de enseñanza son equivalentes cuando se trata de alcanzar objetivos simples como la adquisición y la comprensión de conocimientos. Sin embargo, los métodos de enseñanza centrados en el estudiante conducen a alcanzar objetivos relacionados con la memorización a largo plazo, el desarrollo del pensamiento, el desarrollo de la motivación y el transfer (sic) de aprendizajes.

Entre los métodos centrados en el estudiante, es quizás el aprendizaje en grupo uno de los más utilizados en la actualidad ya que sitúa al alumno en el centro de su propia formación como un actor más en los procesos de adquisición de competencias. Dentro de este modelo se pueden diferenciar dos variantes, el aprendizaje cooperativo y colaborativo, ambos aparecen como una alternativa frente a una enseñanza tradicional. Para algunos autores se trata casi de sinónimos ya que ambos conceptos engloban el hecho del trabajo en grupo con la participación de todos los miembros de este, mientras que nosotros nos posicionamos en la línea de aquellos estudiosos que defienden las diferencias entre ambos modelos, yendo más allá de que el cooperativo no sea más que una subcategoría del colaborativo. De esta forma, Millis y Cottel (1998, citados por García, Robles y Correyero, 2016) sostienen que el aprendizaje cooperativo y colaborativo están situados en un continuo que va de lo más estructurado (cooperativo) a lo menos estructurado (colaborativo). Bruffee (1995, como se citó en García, Robles y Correyero, 2016) defiende que la meta del aprendizaje cooperativo es trabajar juntos en armonía y apoyo mutuo para hallar la solución, mientras que el fin del aprendizaje colaborativo es desarrollar a personas reflexivas, autónomas y elocuentes.

Aunque las fronteras entre ambos tipos de aprendizaje en ocasiones puedan mostrarse difusas, consideramos que, en el caso del proyecto que presentamos, la metodología que empleamos se enmarca dentro del aprendizaje cooperativo que "exige que los estudiantes trabajen juntos en una tarea común, compartan información y se apoyen mutuamente" (Barkley, Cross y Major, 2007, p. 18).

En esta modalidad el profesor planifica las tareas, controla los tiempos, proporciona los materiales necesarios y supervisa la participación de los alumnos y su correcto desempeño de la tarea asignada. La base del aprendizaje es la experiencia del mentor,

algo que existe en el coaching, pero con la diferencia de que mientras en este caso el coacher nunca expresa su experiencia al coachee (alumno) quien aprende de su propia experiencia y conocimientos, mientras que en el caso del mentorship, el mentor sí especifica 
su experiencia, conocimiento, consejos y trucos para que el mentee (alumno) aprenda y pueda continuar su camino sin ayuda del mentor en cuanto tenga la base de conocimiento suficiente (Zamora y Bello, 2011, p. 31).

Onrubia (2005, p. 6) considera que la existencia de una actividad conjunta no exige la presencia ni en el espacio ni en el tiempo de los participantes en la situación,

profesor $\mathrm{y}$ alumnos pueden hacer $\mathrm{y}$ hacen determinadas cosas conjuntamente, aunque lo hagan de manera remota y asíncrona. Dicho de otro modo, lo que hace que la actividad sea efectivamente conjunta no es la copresencia física de los participantes, sino el hecho de que profesor y alumnos actúan el uno para el otro y entre sí, de manera que las actuaciones de cada participante solo se entienden y cobran significado en el marco de, y en referencia a, las actuaciones del resto de participantes.

Pasamos, de este modo, a un modelo de aprendizaje mixto o semipresencial, concepto que nace de la hibridación entre el aprendizaje tradicional basado en la presencialidad y el desarrollo de la educación a distancia mediante herramientas TIC. Esta modalidad mixta de aprendizaje ha tomado otros nombres como hybrid model (Marsh, Mcfadden y Price, 2003) o enseñanza semipresencial (Bartolomé, 2002) y enfatiza la utilidad de lo aprendido, rasgo esencial en nuestro proyecto de innovación docente.

Hinojo, Aznar y Cáceres (2009) describen las principales características diferenciadoras de esta metodología didáctica, como la combinación de sesiones presenciales con sesiones a distancia, estableciendo vínculos entre los participantes y desarrollando habilidades y actitudes que difícilmente se trabajan en experiencias elearning; el refuerzo del aprendizaje con estrategias como juego de roles, prácticas, etc.; la mayor eficiencia por parte de los estudiantes en la resolución de problemas en el trabajo en comparación con los que estudian bajo el aprendizaje electrónico puro. En definitiva, el modelo mixto de enseñanza pretende facilitar el aprendizaje de los estudiantes, contribuir a la comunicación de los alumnos con el docente y de los alumnos entre sí, y, por supuesto, mejorar su nivel de conocimientos como usuarios de las TIC (Santibáñez, 2010).

Por su parte, el aprendizaje móvil "comporta la utilización de tecnología móvil, sola o en combinación con cualquier otro tipo TIC a fin de facilitar el aprendizaje en cualquier momento y lugar" (UNESCO, 2013, p. 6). Tiene unas características que lo hacen único como son la portabilidad, inmediatez, conectividad, ubicuidad y adaptabilidad (Cantillo, Roura y Sánchez, 2012). Cabe sumar a estas características la alta penetración de los dispositivos móviles en el mercado.

Por estas mismas características ya mencionadas, los teléfonos móviles se están convirtiendo en la principal plataforma de desarrollo de otra tecnología de la información y la comunicación emergente, la realidad aumentada. Bajo este concepto "se agrupan aquellas tecnologías que permiten la superposición, en tiempo real, de imágenes, marcadores o información generada virtualmente, sobre imágenes del mundo real"' (Fundación Telefónica, 2011, p. 10).

Los códigos QR almacenan información y la ofrecen de forma rápida a los usuarios que dispongan de un lector de códigos. Fueron creados en 1994 y más tarde estandarizados en 2000 en el entorno industrial para facilitar la localización y gestión de materiales. Estos códigos han ido expandiéndose a otros ámbitos como la mercadotecnia, publicidad, educación, etc., gracias a las funcionalidades de dispositivo de lectura que ofrecen los teléfonos móviles inteligentes.

Estos modelos de aprendizaje y herramientas TIC confluyen en el proyecto que detallamos en el siguiente punto, componiendo un entorno de aprendizaje más cercano y, por tanto, potencialmente más efectivo, a la realidad personal y social del alumno caracterizada por una integración permanente entre lo presencial y lo virtual, en lo que Antonio Bartolomé (2008) denomina como blended lives.

\section{MATERIAL Y MÉTODO}

El proyecto se ha desarrollado en una serie de fases: en primer lugar, se llevó a cabo una recogida de datos, concretamente el estudio de detección de necesidades de aprendizaje mediante la elaboración de una encuesta que fue difundida entre el alumnado y profesorado. La información que se obtuvo aludía, por un lado, a la utilización de equipos audiovisuales en laboratorios y, por otro, a la relación directa de las materias con la comunicación oral. Tras el análisis de los planes de estudio y la asignación de recursos y laboratorios definimos nuestra población diana y, por tanto, la muestra a estudiar se concretó en alumnos y docentes de más de cincuenta asignaturas distribuidas en los tres grados implicados.

Se diseñó un cuestionario de evaluación de los videotutoriales siguiendo una escala de Likert para los alumnos y profesores con la intención de detectar 
la conveniencia de implementar esta herramienta de aprendizaje en la Facultad de Ciencias de la Comunicación. En él, se utilizaron esencialmente ítems dicotómicos, basados en escalas impares, de tipo acuerdo-desacuerdo en cinco posiciones, posibilitando la neutralidad en la opinión de tal modo que el 1 correspondía a "totalmente en desacuerdo", el 2 "moderadamente en desacuerdo", el 3 "ni de acuerdo ni en desacuerdo", el 4 "moderadamente de acuerdo" y el 5 al "estoy totalmente de acuerdo".

El cuestionario constaba de diez ítems para conocer si los alumnos utilizaban estas herramientas de aprendizaje por su cuenta, para qué materias, si lo hacían mediante la lectura de códigos QR y para qué asuntos. También se les preguntó sobre el conocimiento técnico previo al acceso a los laboratorios, en el aula plató y en la de radio, así como su opinión sobre la conveniencia de disponer de videotutoriales que expliquen esos contenidos previamente al desarrollo de las clases. En el mismo cuestionario y mediante un enlace a los vídeos ya realizados, se les pedía a los alumnos su opinión sobre los mismos, si los utilizarían y si los recomendarían a sus compañeros.

La encuesta a los profesores constaba de cinco ítems para conocer su opinión sobre la utilización de los videotutoriales como herramienta de apoyo a la docencia y el aprendizaje de los alumnos, además de otros tres más para que, una vez visionados dos ejemplos prácticos, ofrecieran su punto de vista sobre la conveniencia de implementarlos en las aulas y laboratorios.

Una vez obtenida la certeza de las necesidades formativas de los alumnos y para dar respuesta a esta demanda iniciamos una segunda fase de elaboración y publicación de los recursos audiovisuales. Para ello utilizamos una metodología cooperativa, que pretende, implicar a los alumnos en el proyecto para un óptimo aprendizaje que se inicia con la motivación y selección de alumnos voluntarios en el marco de las asignaturas mencionadas. Continuamos con la elección de las materias que conforman los tutoriales seguido de las fases de preproducción (guion y planificación de rodaje), producción (grabación y rodaje) y posproducción (edición y montaje). Tras la formación de grupos con los alumnos voluntarios, cada uno de éstos eligió, según su propio criterio, un videotutorial a realizar.

Con respecto al plano formal, se utilizaron técnicas de discusión colaborativas para determinar el formato en cuanto a su estilo narrativo, duración, composición, grafismo, etc. Para la toma de decisiones en esta fase de preproducción se tuvo en cuenta las condiciones de visionado del usuario potencial. Al tratarse de un acceso, principalmente, a través de códigos $\mathrm{QR}$ en el espacio formativo mediante dispositivos móviles, el tutorial debía ser conciso, breve y directo. El formato elegido es un tutorial de una duración entre uno y dos minutos con una realización simple y cuidada donde predominan los primeros planos $\mathrm{y}$ en el que un narrador extradiegético conduce mediante voz en off $\mathrm{y}$ subtítulos opcionales la acción formativa.

El medio elegido para emplazar los vídeos formativos es YouTube, como principal plataforma de vídeos (Alexa, 2016). YouTube permite no solo el acceso y difusión a los alumnos del centro sino también pretende servir de ayuda a un público global con similares necesidades formativas. Para aumentar dicho público potencial hemos realizado versiones en un segundo idioma, concretamente en inglés, disponibles mediante un acceso directo sobreimpresionado, tipo etiqueta, en los primeros instantes del vídeo en castellano. La accesibilidad de colectivos con limitaciones sensoperceptivas también está prevista a través de los subtítulos ya mencionados y versiones con sobreimpresiones de intérpretes de lenguaje de signos.

Se creó un canal llamado 'Proyecto Innovación Docente (PIE) Ciencias de la Comunicación UMA' en el que se puso a disposición del público los materiales formativos de forma independiente o bien agrupados en listas de reproducción que facilitan al usuario el acceso a contenidos relacionados. Las listas de reproducción creadas se denominan «Inside TV», que agrupa los videotutoriales referidos a equipamiento de plató de televisión; «Inside Photography», que aglutina a los relacionados con el estudio fotográfico; «Radio Studio», que incluye vídeotutoriales de equipamiento y operación en el estudio de radio y, por último, «Speech In», que agrupa vídeos formativos relacionados con técnicas de comunicación.

Tabla 1. Contenido de los subcanales de YouTube

\begin{tabular}{cccc}
\hline $\begin{array}{c}\text { La cámara } \\
\text { de estudio }\end{array}$ & $\begin{array}{c}\text { Inside } \\
\text { Photography }\end{array}$ & $\begin{array}{c}\text { Radio } \\
\text { Studio }\end{array}$ & Speech In \\
\hline $\begin{array}{c}\text { El } \\
\text { mezclador } \\
\text { de vídeo }\end{array}$ & $\begin{array}{c}\text { La cámara } \\
\text { fotográfica }\end{array}$ & $\begin{array}{c}\text { El } \\
\text { primer } \\
\text { contacto }\end{array}$ & $\begin{array}{c}\text { Hablar en } \\
\text { público (1) }\end{array}$ \\
\hline $\begin{array}{c}\text { El } \\
\text { servidor } \\
\text { de vídeo }\end{array}$ & $\begin{array}{c}\text { Esquema de } \\
\text { iluminación } \\
\text { básico }\end{array}$ & $\begin{array}{c}\text { Las } \\
\text { señales } \\
\text { de radio }\end{array}$ & $\begin{array}{c}\text { Hablar en } \\
\text { público (2) }\end{array}$ \\
\hline $\begin{array}{c}\text { La } \\
\text { tituladora }\end{array}$ & El foco & & $\begin{array}{c}\text { Citas } \\
\text { bibliográficas }\end{array}$ \\
\hline \multicolumn{4}{c}{ El } \\
\end{tabular}


A partir de la URL o dirección web de cada vídeo se generaron los códigos QR a través de www.codigos-qr.com. Una vez obtenidos los códigos se ubicaron en los espacios formativos mediante carteles y pegatinas, según el caso. En cuanto a la propiedad intelectual de los materiales generados y siguiendo la filosofía colaborativa y de acceso abierto del proyecto, optamos por licencias Creative Commons en su modalidad de reconocimiento (attribution) que permite la explotación libre en todas sus facetas con la simple obligación de citar el origen.

\section{ANÁLISIS Y RESULTADOS}

En este apartado se ofrecerán las principales conclusiones del estudio, en función con los datos obtenidos y la discusión realizada.

Una vez terminada la fase de recogida de información se procedió a analizar las respuestas a la encuesta dirigida a los alumnos y profesores. En concreto participaron 108 alumnos y 24 profesores de todos los cursos de los tres grados impartidos en el Centro. Por decisión del equipo, los cuestionarios se realizaron de forma anónima por lo que no podemos describir la muestra en cuanto a género y edad. El diseño de la investigación, de carácter cualitativo, es no experimental, transversal y descriptivo de tipo comparativo. El primer cuestionario, dirigido a los alumnos, tras ser analizado con el software SPSS, ofrece un coeficiente de fiabilidad (Alfa de Cronbach) de 0,705 mientras que el cuestionario de los profesores obtiene un coeficiente de 0,707 .

En cuanto al contenido, la primera cuestión planteaba si los alumnos estaban habituados a utilizar los videotutoriales como herramienta de aprendizaje de manera autónoma, por su cuenta. La respuesta fue afirmativa en un 56\% de los alumnos. Respecto a su contenido estaba mayoritariamente relacionado con el uso de las TIC $(65,7 \%)$, seguido de contenidos de ocio $(37,3 \%)$.

En cuanto al conocimiento previo del manejo del software y de los equipos en los laboratorios, el grado de desconocimiento es menor que en el resto de instalaciones (20\%), conformándose una mayoría con conocimiento moderado o total $(50 \%)$.

Este mayor conocimiento en el uso de los laboratorios contrasta, en general, con el que manifestaron respecto a los equipos del aula plató y, sobre todo, del aula de radio donde se alcanzan cotas de desconocimiento muy superiores. Concretamente, el $42,5 \%$ de los alumnos reconocieron un total desconocimiento o un moderado desconocimiento en el manejo de equipo en el aula plató frente al 29,3\% que señaló un total o moderado conocimiento. En el aula de radio, el grado de desconocimiento era todavía superior situándose en el $54,7 \%$ de los encuestados, frente al $22,6 \%$ que señalaron su total o moderado conocimiento.

No es por ello extraño que los alumnos muestren mayoritariamente $(90,2 \%)$ la conveniencia de disponer de videotutoriales que expliquen con antelación el manejo de los equipos en dichos espacios. Lo mismo que para mostrar las técnicas de comunicación oral como hablar en público o de expresión $(71,1 \%)$.

Todo ello nos lleva a concluir, tras esta primera encuesta de detección de necesidades, que la utilización de los videotutoriales como herramienta para el aprendizaje es percibida como necesaria por parte de los alumnos de los tres grados de Ciencias de la Comunicación en un elevado porcentaje.

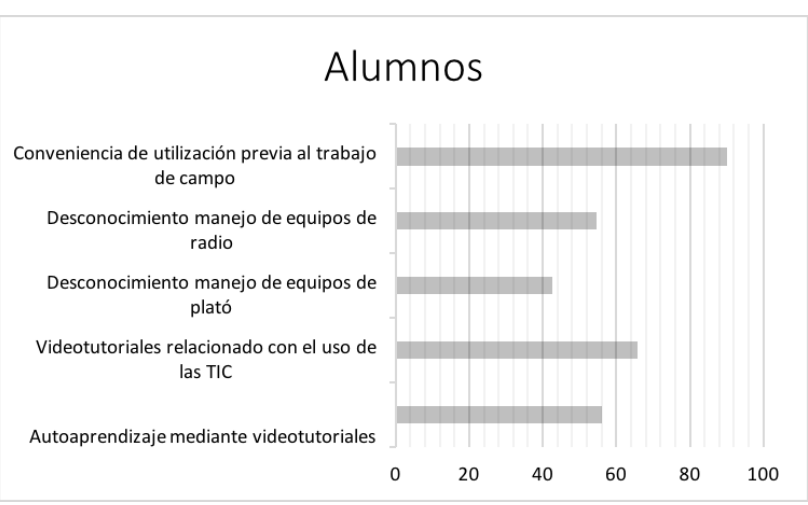

Figura 2. Respuesta de alumnos a la encuesta sobre el uso de videotutoriales en los laboratorios de Ciencias de la Comunicación

En cuanto al punto de vista de los profesores respecto a la conveniencia de disponer de esta herramienta, resulta también mayoritaria su opinión a favor $(95,7 \%)$ y que debería disponerse previamente al trabajo en los espacios de laboratorios y platós $(91,3 \%)$.

$\mathrm{El}$ aspecto en el que el profesorado muestra dudas es sobre si los videotutoriales deberían ser realizados por los propios alumnos como herramienta de aprendizaje $(49,1 \%)$ frente al $30,4 \%$ que están totalmente o moderadamente en desacuerdo. 


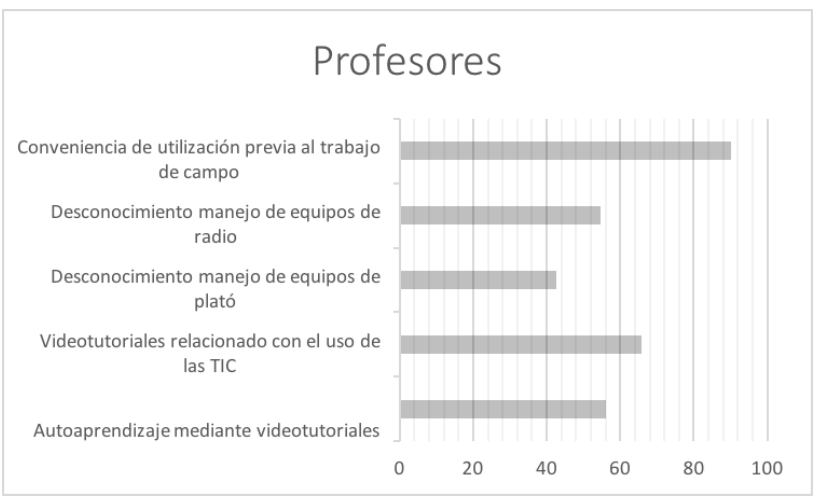

Figura 2. Respuesta de profesores a la encuesta sobre el uso de videotutoriales en los laboratorios de Ciencias de la Comunicación

\section{DISCUSIÓN Y CONCLUSIONES}

En el momento de la redacción del presente artículo, el canal PIE Ciencias de la Comunicación UMA, creado en febrero de 2016 en modo de pruebas y hecho público en julio del mismo año, se encuentra en su primera fase de implementación. A partir del análisis de los datos obtenidos a través de una encuesta dirigida a una muestra de estudiantes y profesorado de los tres grados impartidos en la Facultad de Ciencias de la Comunicación de la Universidad de Málaga y, tras comenzar la experimentación con los videotutoriales asociados a códigos QR como herramienta didáctica, consideramos su pertinencia para mejorar la asimilación de los contenidos objeto del aprendizaje, en el caso de los estudiantes que participaron en su elaboración, y así mismo, se facilita el recuerdo de estos contenidos en el caso de los estudiantes usuarios. Creemos pues, que tan importante es la audiencia y utilidad del videotutorial en sí, como el aprendizaje cooperativo experimentado por los alumnos y profesorado participantes en el proyecto.

Respecto al porcentaje $(30,4 \%)$ de profesorado encuestado del Centro que duda sobre si los videotutoriales deberían ser realizados por los tutores o por los propios alumnos, creemos que muestran una baja predisposición a implementar esta metodología como herramienta de aprendizaje, cuestión que contrasta con nuestro punto de vista sobre la importancia de la actividad y el contexto, que coincide con el de Tirado, Méndez y Aguaded (2008, p. 166) para quienes "el conocimiento es parte y producto de la actividad, el contexto y la cultura en que se desarrolla y utiliza", en definitiva que "aprender y hacer son acciones inseparables". En el caso de este proyecto, se conjugan elementos TIC, con aspectos del blended learning, la formación in situ y el aprendizaje colaborativo, con el objetivo de convertir al alumno en protagonista de su propio aprendizaje para que éste sea significativo.

Será, por tanto, una labor indispensable y prevista en el cronograma del proyecto la celebración de unas jornadas divulgativas, dirigidas a profesores, en las que se presenten los contenidos y resultados del mismo, así como el impacto positivo sobre la comunidad educativa de la Facultad, para involucrar a ese profesorado que un principio se haya mostrado escéptico ante la herramienta.

El PIE "Recursos tecnológicos para la enseñanza en laboratorios audiovisuales: videotutoriales y códigos QR" tiene una vocación universal y colaborativa y por ello en su nueva fase pretende añadir a los videotutoriales ya realizados en español e inglés, nuevos contenidos en lengua de signos y audiodescripción con la intención no solo de alcanzar a una audiencia variada, sino además para sensibilizar a los comunicadores del futuro de la importancia de incluir a todos los colectivos en sus productos informativos.

Aunque el proyecto de innovación docente tiene prevista su finalización en mayo de 2017, momento en el que podremos realizar un exhaustivo análisis de sus resultados globales, tanto cuantitativos mediante herramientas de analítica web como cualitativos a través de encuestas de satisfacción, se puede avanzar nuestra valoración altamente positiva y la vez muy instructiva, tanto por el feedback recibido hasta el momento, como por nuestra observación directa de su impacto en las aulas.

\section{REFERENCIAS}

Alexa Web Information Company. (2016). Top sites. Recuperado de http://www.alexa.com/topsites (2016-09-15)

Barkley, E., Cross, K. y Major, C. (2007). Técnicas de aprendizaje colaborativo: Manual para el profesorado universitario. Madrid: Morata.

Bartolomé, A. (2002). Universidades en la red. ¿Universidad presencial o virtual? Crítica, LII 896, 34-38.

Bartolomé, A. (2008). Entornos de aprendizaje mixto en educación superior. Revista Iberoamericana de Educación a Distancia, 11(1), 15-51.

Bravo, J. (1996). ¿Qué es el vídeo educativo?". Comunicar, nº 6, 100-105.

Brazuelo, F. y Cacheiro, M. (2010). Diseño de páginas webs educativas para teléfonos móviles. Edutec. Revista Electrónica de Tecnología Educativa, 32, 1-14.

Cabero, J., Gisbert, M. (2005). La formación en internet: Guía para el diseño de materiales formativos. Sevilla: Mad.

Cantillo, C., Roura, M. \& Sanchez, A. (2012). Tendencias actuales en el uso de dispositivos móviles en educación. LaEduc@ción Digital Magazine, 147, 1-20.

Cebrián, M. (1987). El vídeo Educativo. II Congreso de Tecnología Educativa. Madrid: Sociedad Española de Pedagogía.

Espuny, C., Gonzalez, J., Lleixa, M. y Gisbert, M. (2011). Actitudes y expectativas del uso educativo de las redes 
sociales en los alumnos universitarios. El impacto de las redes sociales en la enseñanza y el aprendizaje. Revista de Universidad y Sociedad del Conocimiento, 1 (8), 171185.

Fernández, A. (2007). Nuevas metodologías docentes. Instituto de Ciencias de la Educación. Universidad Politécnica de Valencia.

Fundación Telefónica. (2011). Realidad aumentada: una nueva lente para ver el mundo. Madrid: Fundación Telefónica.

Garcia, I., Robles, C. y Correyero, B. (2016). La gestión de equipos de trabajo y productos radiofónicos en las radios universitarias. Revista Latina de Comunicación Social, 71, 696-714.

Greene, K. (2007). Practical holographic video. Massachusetts: Technology review.

Gómez, B. Tapia, F. (2011): Facebook y Tuenti:de plataforma de ocio a herramienta e-learning. Prisma Social: Revista de investigación social, (6) 238-260

Hinojo, F., Aznar, I. \& Caceres M. (2009). Percepciones del alumnado sobre el blended learning en la Universidad. Comunicar. Revista Científica de Educomunicación, (33), 165-174.

Jiménez, D. y Marín, G. (2012). Asimilación de contenidos y aprendizaje mediante el uso de videotutoriales. Enseñanza \& Teaching: Revista Interuniversitaria de Didáctica (30), 63-79.

Llanos, F. (2008). Videoredada. Recuperado de http://videoredada.blogspot.com.es/2008/02/historia-delvideo-para-internet_13.html (2016-09-01).

López, Ábalos y Espejo (2012). Sistema basado en procesamiento de lenguaje natural para apoyo al aprendizaje de alumnos universitarios. Relada 6(2): 206-214.

Mackey, T. \& Ho, J. (2008). Exploring the relationships between web usability and students' perceived learning in webbased multimedia (wbmm) tutorials. Computers \& Education, (50), 386-409.

${ }^{1}$ Según Mauri, T., Coll, C. y Colomina, R. (2005) se presentan en formato multimedia o hipermedia y su utilización se prevé que sea posible en situaciones en que el grado de coincidencia física, espacial y temporal de profesores y alumnos pueda darse en un mínimo o incluso no existir. Ver http://www.um.es/ead/red/M2/mauri_onrubia.pdf

2 Se pueden consultar, entre otras, las experiencias descritas por Gómez, B. Tapia, F. (2011): "Facebook y Tuenti: de plataforma de ocio a herramienta e-learning". Prisma Social: revista de investigación social, ISSNe 1989-3469, No. 6, págs. 238-260

${ }^{3}$ Según Tesouro, M. y Puiggalí, J. es aquella escuela que ni imparte docencia dentro de un único espacio físico (escuela) ni dentro de unos horarios fijos (horario escolar)
Marse, B. (2012). YouTube: las claves para aprovechar todas sus potencialidades. Barcelona: Profit Editorial.

Marsh, G., Mcfadden, A. y Price, B (2003). Blended instruction: Adapting conventional instruction for large classes. Online Journal of Distance Learning Administration, 4(6).

Mauri, T., Onrubia, J., Coll, C. y Colomina, R. (2005). La calidad de los contenidos educativos reutilizables: Diseño, usabilidad y prácticas de uso. Red. Revista de Educación a Distancia, (50), 1-9.

Mayer, R. (2001). Multimedia learning. New York: Cambridge University Press.

Onrubia, J. (2005). Aprender y enseñar en entornos virtuales: Actividad conjunta, ayuda pedagógica y construcción del conocimiento. Red. Revista de educación a distancia, (2), $1-16$.

Polo, V. (2010): Juzgado didáctico: tránsito de la práctica jurídica del aula a la realidad del ejercicio profesional. En de Castro, A. (ed): Innovar para educar: prácticas universitarias exitosas. Barranquilla: Ediciones Uninorte. 60-70.

Santibáñez, J. (2010). Aula virtual y presencial en aprendizaje de comunicación audiovisual y educación. Comunicar. Revista científica de educomunicación, (35), 183-191.

Tirado, R; Mendez, J.M. \& Aguaded, J.I. (2008). El proyecto Drog@: Comunidades virtuales de aprendizaje. Comunicar, (30), 165-169.

UNESCO. (2013). Directrices de la UNESCO para las políticas de aprendizaje móvil. París: UNESCO.

Zamora, J. \& Bello, S. (2011). La videocreatividad, el m-learning y los blogs como herramientas de innovación en el ámbito educativo universitario. Revista Electrónica de Investigación, Docencia y Creatividad, (1), 28-45.

y en ella cualquier alumno o profesor puede conectarse e interactuar sin restricciones espaciales ni temporales. Ver Gómez, B. Tapia, F (2011).

4 Estos autores consideran contenidos educativos reutilizables interactivos aquellas actuaciones que un alumno desarrolla individualmente accediendo a la propuesta de contenidos para resolver una tarea de aprendizaje. El profesor o tutor no está presente, pero lo que el alumno realiza tiene relación con lo que ha ocurrido anteriormente en el marco del diseño de los contenidos incluidos en los materiales y puede, según las condiciones educativas establecidas, tener relación con lo que vaya a ocurrir más tarde, en situaciones de valoración de los resultados de aprendizaje o en las posibilidades establecidas de progreso del alumno. 